\title{
Effect of negative-pressure wound therapy on open fractures of the lower limb
}

Janna Joethy ${ }^{1}$, MBBS, MMed, Sandeep J Sebastin ${ }^{2}$, MMed, FAMS, Alphonsus Khin Sze $\underline{\text { Chong }}^{2}$, MBBS, MRCS, Yeong Pin Peng ${ }^{2}$, MBBs, frcs, Mark E $\underline{\text { Puhaindran }}^{2}$, MBBs, Mrcs

INTRODUCTION Early debridement and coverage has long been regarded as the standard of care for open fractures of the lower limb, as infection is a serious complication. However, the best time for wound closure remains controversial. Negative-pressure wound therapy (NPWT) is thought to result in reduced flap infection and failure. To determine the effect of NPWT, we reviewed patients with open fractures of the lower limb and compared the rates of infection and flap failure in two time-based cohorts.

METHODS Two cohorts of patients (periods 2003-2004 and 2008-2009) with Gustilo type IIIB open tibial fractures were recruited and their outcomes were compared. In the 2003-2004 cohort, wounds were dressed with occlusive dressing. In the 2008-2009 cohort, all patients underwent NPWT. Data was retrospectively analysed with regard to infection, failure, age, type of flap, comorbidities and defect size. The incidences of infection and flap failure were further analysed for any statistical difference between the different treatment protocols.

RESULTS In the 2003-2004 cohort, 33\% of patients developed infection and $11 \%$ had flap failure. However, in the 2008-2009 cohort, $10 \%$ of patients developed infection and $6 \%$ had flap failure. The difference in the incidence of infection was statistically significant between the two cohorts $(p=0.029)$.

CONCLUSION Patients in the 2008-2009 cohort had better outcomes, and we are of the opinion that performing NPWT may have contributed to this result.

Keywords: free flap, negative-pressure wound therapy, occlusive dressing, open tibial fractures, vacuum-aided closure

\section{INTRODUCTION}

The optimal time for the management of wounds has evolved from the 'golden period' of wound closure within six hours, to early wound coverage as advocated by Godina. ${ }^{(1)}$ Although these concepts were initially thought of as dogma, some aspects of these concepts have persisted. However, subsequent work in this field has not defined the best time period for wound coverage.

More recent in the evolution of wound management has been the advent of negative-pressure wound therapy (NPWT), with initial work on NPWT done by Morykwas et al. ${ }^{(2)}$ While there are numerous publications on the benefits of NPWT, there is only one Level 1 randomised study on it. ${ }^{(3)}$ We therefore performed a cohort study to assess the management of open tibial fractures at our institute, in order to determine whether there was any difference in the outcome and infection rates of patients before and after the introduction of NPWT. We anticipated that patients with open tibial fractures managed using NPWT would have lower infection and flap failure rates than those managed using occlusive dressing alone.

\section{METHODS}

A retrospective study of patients who underwent free flap reconstruction for lower limb open fractures at the Department of Hand and Reconstructive Microsurgery, National University Health System, Singapore, was conducted. Two cohorts of patients from the periods 2003-2004 ( $\mathrm{n}=18$ ) and 2008-2009 ( $\mathrm{n}=51$ ) were identified. In the 2003-2004 cohort, wounds were dressed with occlusive dressing, whereas in the 2008-2009 group, all patients underwent NPWT. NPWT was applied at $125 \mathrm{mmHg}$ and in the continuous mode, and dressings were changed every three to four days, depending on the amount of exudate. The hospital's database was used to identify patients for whom free flap reconstructions were performed for open fractures in the lower limb during the aforementioned time period. All surgeries were performed by senior surgeons at our centre.

Data on patients who underwent free flap reconstruction for Gustilo type IIIB traumatic lower third defects was extracted. Only fractures of the tibia were included, and patients with other or additional fractures were excluded from the study. Several parameters were drawn and noted from our data age of patient, location and size of defect, and any significant comorbidity. The wound area, typically recorded as length and breadth measurements, was calculated as length $\times$ breadth. Type of flap, time from injury to wound coverage, and the duration of flap surgery were also recorded. Patient records were checked to identify flap infection and flap failure

\footnotetext{
${ }^{1}$ Department of Plastic, Reconstructive and Aesthetic Surgery, Singapore General Hospital, ${ }^{2}$ Department of Hand and Reconstructive Microsurgery, National University Health System, Singapore

Correspondence: Dr Mark E Puhaindran, Consultant, Department of Hand and Reconstructive Microsurgery, National University Health System, 1E Kent Ridge Road, NUHS Tower Block, Level 11, Singapore 119228. Mark_E_Puhaindran@nuhs.edu.sg
} 
Table I. Summary of results.

\begin{tabular}{|c|c|c|}
\hline \multirow[t]{2}{*}{ Variable } & \multicolumn{2}{|c|}{ No. of patients (\%) } \\
\hline & $\begin{array}{c}2003-2004 \\
(n=18)\end{array}$ & $\begin{array}{c}2008-2009 \\
(n=51)\end{array}$ \\
\hline Age* (yrs) $^{*}$ & 38 & 40 \\
\hline Pre-existing morbidities & $2(11)$ & $6(12)$ \\
\hline Duration of free flap surgery* & 5 hrs 55 mins & $5 \mathrm{hrs} 35 \mathrm{mins}$ \\
\hline Time to flap surgery ${ }^{+}$(days) & 16.8 & 10.8 \\
\hline Defect size ${ }^{+}\left(\mathrm{cm}^{2}\right)$ & 166 & 192 \\
\hline $\begin{array}{l}\text { Type of free flap } \\
\text { Gracilis } \\
\text { Latissimus dorsi } \\
\text { Lateral arm } \\
\text { Anterolateral thigh }\end{array}$ & $\begin{array}{l}7(39) \\
5(28) \\
4(22) \\
2(11)\end{array}$ & $\begin{array}{c}25(49) \\
19(37) \\
3(6) \\
4(8)\end{array}$ \\
\hline Use of NPWT & $0(0)$ & $51(100)$ \\
\hline Clinical infection & $6(33)$ & $5(10)$ \\
\hline Flap failure & $2(11)$ & $3(6)$ \\
\hline
\end{tabular}

${ }^{*}$ Data is expressed as median. ${ }^{\dagger}$ Data is expressed as average.

NPWT: negative-pressure wound therapy

rates. Infection was only recorded if it was confirmed by documentation by the surgical team. Statistical analysis to evalutate any difference between variables was performed using Fisher's test. The number of days to surgery and the outcome of the flaps were also assessed. Approval for the study was not obtained as no identifiable data was retrieved.

\section{RESULTS}

The average age of the patients in the 2003-2004 cohort was 38 (range 19-60) years, whereas that of the 2008-2009 cohort was 40 (range 19-78) years. The median time taken for free flap surgery in the 2003-2004 cohort was 5 hrs 55 mins (range 3 hrs 45 mins-10 hrs 30 mins). In the 2008-2009 cohort, the median duration of free flap surgery was 5 hrs 35 mins (range 2 hrs 15 mins-10 hrs 30 mins). The upper limits of the aforementioned ranges represent a small number of cases of fixation and flap surgery. In the 2003-2004 cohort, 7 (39\%) flaps were free gracilis flaps, 5 (28\%) were free latissimus dorsi flaps, 4 (22\%) were free lateral arm flaps and $2(11 \%)$ were free anterolateral thigh flaps. In the 2008-2009 cohort, 25 (49\%) flaps were free gracilis flaps, 19 (37\%) were free latissimus dorsi flaps, 3 (6\%) were free lateral arm flaps and $4(8 \%)$ were free anterolateral thigh flaps. The average defect size of the 2003-2004 cohort was $166 \mathrm{~cm}^{2}$, while that of the 2008-2009 cohort was $192 \mathrm{~cm}^{2}$. The average time to flap surgery was 16.8 (range 1-62) days in the 2003-2004 cohort, while that in the 2008-2009 cohort was 10.8 (range $1-60)$ days. A total of $2(11 \%)$ patients in the 2003-2004 cohort had pre-morbid conditions. In the 2008-2009 cohort, 6 (12\%) patients had a range of pre-existing morbidities such as hypertension, diabetes mellitus, asthma and ischaemic heart disease. A summary of our results is presented in Table I.

Flap failure (either partial or total) was noted in $2(11 \%)$ and $3(6 \%)$ patients in the 2003-2004 and 2008-2009 cohorts, respectively. Clinical flap infection was respectively seen in
$6(33 \%)$ and $5(10 \%)$ patients in the 2003-2004 and 2008-2009 cohorts. Two-tailed Fisher's exact test showed that the difference between the incidences of infection of the two cohorts was significant $(p=0.0288)$. However, the difference between the incidences of flap failure of the two treatment groups was not statistically significant $(p=0.598)$.

\section{DISCUSSION}

The six-hour window to operative debridement of open fractures of the lower limb was initially thought to be crucial in the reduction of infection rates, with this period of time often referred to as the golden period for wound repair. Although the precise origin of this six-hour rule is unknown, its mention dates back to 1898 , following a study by Friedrich. ${ }^{(4)}$ Based on the study by Friedrich, the optimal time for suturing wounded soldiers during the Second World War was suggested to be within six hours after trauma. ${ }^{(5)}$ Nevertheless, regardless of the origin of the six-hour rule, debridement within six hours of injury is a widely accepted standard of care for open fractures, ${ }^{(6)}$ though some authors have reported no benefit from debridement within the golden period..$^{(7-10)}$

In 1986, Godina established a dictum that encouraged the early reconstruction of complex trauma of the extremities. ${ }^{(1)}$ According to Godina's rule, a "proper debridement [is to be] done immediately after injury". He found that free flap transfer within 72 hours of trauma increased flap survival, reduced infection and shortened the hospital stay of patients. Godina suggested that there was a short 'safe window' immediately after sustainment of trauma for flap transfer, following which the risks increase. Conversely, Yaremchuk et al recommended that flaps be transferred between 7 and 14 days after injury, and after serial debridement. ${ }^{(11)}$ The argument in favour of this approach is that the zone of injury, which may often not be apparent at presentation, can be determined through sequential debridements. The ideal timing for free tissue transfer remains controversial. ${ }^{(12)}$ While some authors claim that the timing of wound management has no effect on flap success rates, others have found otherwise. Khouri and Shaw reported that free flap failure was not time-dependent and the most important factor was the magnitude of the injury sustained. ${ }^{(13)}$

Early work by Morykwas et al led to the advent of NPWT, which is now in widespread clinical use. ${ }^{(2)}$ However, to the best of our knowledge, the association between infection and NPWT had not been established until 2011. ${ }^{(6)}$ The present study was therefore performed to evaluate the effect of NPWT on the incidence of infection and flap failure in patients with open fractures of the lower limb who underwent free flap reconstruction of the lower limb.

We found that the average ages of the patients from the two cohorts in our study were similar (38 years for the 2003-2004 cohort and 40 years for the 2008-2009 cohort). The 2008-2009 cohort had a slightly shorter median operating time. The type of flap chosen was dependent on wound size 
and vascular status. In both cohorts, the most commonly used flap was the free gracilis flap, followed by the free latissimus dorsi flap. Despite this, there were different rates of infection and flap failure in the two cohorts; the lower rates of infection and flap failure observed in the 2008-2009 cohort may be due to the use of NPWT, which has been reported to increase blood flow to the wound site. ${ }^{(3)}$ While the mechanism of increased angiogenesis conferred by NPWT is unknown, animal studies have shown that wounds treated by negative pressure (i.e. vacuum-assisted closure) showed elongated vessels aligned in parallel and consistent with physiological function, compared to tortuous and disoriented vessels in control wounds that underwent occlusive dressing. ${ }^{(15)}$ Furthermore, in contrast to the generalised hypoxia seen in occlusive dressing-treated control wounds, negative-pressuretreated wounds displayed a well-oxygenated wound bed.

In the present study, the incidence of infection was lower in the 2008-2009 cohort, where 5 (10\%) patients had infections. In contrast, 6 (33\%) patients in the 2003-2004 cohort had infections. The difference in the incidence of infection was statistically significant between the two cohorts, suggesting the potential validity of our hypothesis that NPWT reduces infection. Other authors have similarly found a reduction in the rate of deep infection when NPWT was used for open tibial fracture wounds. ${ }^{(16)}$ There were $2(11 \%)$ and $3(6 \%)$ incidences of flap failure in the 2003-2004 and 2008-2009 cohorts, respectively. The difference in the incidence of flap failure between the two cohorts was not statistically significant in our study.

There were several limitations to our study. First, as a retrospective cohort study, it had inherent drawbacks. However, while conducting a randomised prospective study would have been ideal, it is difficult to perform, as most physicians already use NPWT for the management of open fractures of the lower limb despite a paucity of Level 1 evidence. ${ }^{(3)}$

Second, the two cohorts in our study were chosen to determine the effect of management with NPWT on the outcome of patients with traumatic wounds. However, it was difficult to directly compare the two cohorts for various reasons. For instance, although all surgeries in the two cohorts were performed by senior surgeons, the level of surgical expertise may have varied. There were also more flaps performed in the 2008-2009 cohort, perhaps reflecting the learning curve of the surgeons involved, with the number of flaps performed increasing as the surgeons became more adept. On the contrary, Parrett et al reported that free flap transfers were performed less frequently after NPWT became available. ${ }^{(14)}$ They attributed this trend to the availability of NPWT and an improved understanding of the vascular anatomy of the lower leg. In their study, the learning curve was associated with fewer flaps being performed. ${ }^{(14)}$ Apart from this, the population sizes of the two cohorts in our study were dissimilar, making direct comparison difficult. Due to the wide disparity in the number of patients in the two cohorts, an initial attempt was made to perform a matched cohort study with equal numbers of patients in them. However, we found that this approach would result in an easily biased match. Thus, we decided to retain the two patient cohorts as they were, without any matching.

Third, many parameters (e.g. history of smoking, severity of injury and accuracy of wound size) that might have had a bearing on the outcome of our patients were not included for study. However, as the traumatic force contributes to the severity of wounds, we chose to only study tibial fractures so that traumatic force was kept relatively constant.

Despite the limitations listed, the present study found that the management of open tibial fractures with NPWT was associated with favourable results, which is similar to other studies. DeFranzo et al reported that NPWT, when used on exposed bone and tendon, resulted in the formation of granulation tissue over the exposed area and sometimes obviated the need for flap surgery. ${ }^{(17)}$ DeFranzo et al also reported that the use of NPWT reduced tissue oedema, and diminished the circumference and surface area of the wound. ${ }^{(17)}$ Although we did not measure these parameters (i.e. tissue oedema, and circumference and surface area of the wound) in our study, we anecdotally concur that NPWT did indeed reduce tissue oedema. While Godina's study showed the benefit of surgery within 72 hours, ${ }^{(1)}$ Bhattacharyya et al ${ }^{(18)}$ found that NPWT allowed soft tissue cover to be performed within seven days without any increase in infection rate. Our own data from the NPWT cohort (i.e. the 2008-2009 cohort) showed that two of five infections $(40 \%)$ in our series were seen in patients who had surgery after seven days.

Since the introduction of NPWT, we have advocated its use for the management of open tibial fractures. Although we had anecdotal evidence supporting its use in such cases, the purpose of this study was to systematically assess the differences in the outcome of our patients following the introduction of NPWT. In spite of the various inherent difficulties associated with cohort studies of this nature, our results confirm the benefits of NPWT for the management of open tibial fractures of the lower limbs. Although the difference between the incidences of flap failure in the two cohorts was not statistically significant, we are of the view that NPWT helped to reduce flap failure. The use of NPWT was also found to significantly reduce the rate of infection in patients with lower third tibial open fractures.

The management of open fractures of the lower limb varies from centre to centre, and from surgeon to surgeon. Nevertheless, guidelines by BAPRAS (British Association of Plastic, Reconstructive and Aesthetic Surgeons) and BOA (British Orthopaedic Association) have been set up to standardise care for the treatment of open fractures. ${ }^{(19)}$ In National University Hospital, Singapore, patients with open fractures of the lower limb have been jointly managed since 2000. Our results appear to reaffirm that the triple approach involving early and serial 
debridement, combined orthoreconstructive involvement and negative pressure dressing is beneficial for the management of such patients.

\section{REFERENCES}

1. Godina M. Early microsurgical reconstruction of complex trauma of the extremities. Plast Reconstr Surg 1986; 78:285-92.

2. Morykwas MJ, Argenta LC, Shelton-Brown El, McGuirt W. Vacuum-assisted closure: a new method for wound control and treatment: animal studies and basic foundation. Ann Plast Surg 1997; 38:553-62.

3. Stannard JP, Volgas DA, Stewart R, McGwin G Jr, Alonso JE. Negative pressure wound therapy after severe open fractures: a prospective randomized study. J Orthop Trauma 2009; 23:552-7.

4. Friedrich PL. Die aseptische Versorgung frischer Wundern. Langenbecks Arch Klin Chir 1898; 57:288-310. German.

5. Peterson AS. The "Golden Period" for Wound Repair. J Lancaster Gen Hospital 2010; 5:134-5.

6. Pollak AN. Timing of debridement of open fractures. J Am Acad Orthop Surg 2006; 14:S48-51.

7. Bednar DA, Parikh J. Effect of time delay from injury to primary management on the incidence of deep infection after open fractures of the lower extremities caused by blunt trauma in adults. J Orthop Trauma 1993; 7:532-5.

8. Ashford RU, Mehta JA, Cripps R. Delayed presentation is no barrier to satisfactory outcome in the management of open tibial fractures. Injury 2004; 35:411-6.

9. Spencer J, Smith A, Woods D. The effect of time delay on infection in open long-bone fractures: a 5-year prospective audit from a district general hospital. Ann R Coll Surg Engl 2004; 86:108-12.

10. Pollak AN, Jones AL, Castillo RC, et al. The relationship between time to surgical debridement and incidence of infection after open high-energy lower extremity trauma. J Bone Joint Surg Am 2010; 92:7-15.

11. Yaremchuk MJ, Brumback RJ, Manson PN, et al. Acute and definitive management of traumatic osteocutaneous defects of the lower extremity. Plast Reconstr Surg 1987; 80:1-14

12. Baumeister S, Follmar KE, Zenn MR, Erdmann D, Levin LS. Strategy for reoperative free flaps after failure of a first flap. Plast Reconstr Surg 2008; 122:962-71.

13. Khouri RK, Shaw WW. Reconstruction of the lower extremity with microvascular free flaps: a 10-year experience with 304 consecutive cases. J Trauma 1989; 29:1086-94.

14. Parrett BM, Matros E, Pribaz JJ, Orgill DP. Lower extremity trauma: trends in the management of soft-tissue reconstruction of open tibia-fibula fractures. Plast Reconstr Surg 2006; 117:1315-22; discussion 1323-4.

15. Erba P, Ogawa R, Ackermann M, et al. Angiogenesis in wounds treated by microdeformational wound therapy. Ann Surg 2011; 253:402-9.

16. Blum ML, Esser M, Richardson M, Paul E, Rosenfeldt FL. Negative pressure wound therapy reduces deep infection rate in open tibial fractures. J Orthop Trauma 2012; 26:499-505.

17. DeFranzo AJ, Argenta LC, Marks MW, et al. The use of vacuum-assisted closure therapy for the treatment of lower-extremity wounds with exposed bone. Plast Reconstr Surg 2001; 108:1184-91.

18. Bhattacharyya T, Mehta P, Smith M, Pomahac B. Routine use of wound vacuum-assisted closure does not allow coverage delay for open tibia fractures. Plast Reconstr Surg 2008; 121:1263-6.

19. Nanchahal J, Nayagam D, Khan U, et al. Standards for the Management of Open Fractures of the Lower Limb. London: Royal Society of Medicine Press, 2009. 